\title{
A personalized version of teflon felt sandwich technique for acute type a aortic dissection
}

Bektaş Battaloğlư ${ }^{1}$ Barış Akça ${ }^{1}$, Nevzat Erdil ${ }^{1}$, Cengiz Çolak ${ }^{1}$, Olcay M. Dişli ${ }^{1}$,

1) Inonu University Faculty of Medicine Department of Cardiovascular Surgery, MD, Malatya, Turkey

\begin{abstract}
Although, there are important technical developments in surgical repair for acute type A aortic dissection, the surgical intervention still carries some difficulties especially when the aortic dissection spreads to the sinuse of valsalva. Here, a modified version of the felt sandwich technique is described in order to support the entire aortic root externally and overcome bleeding without causing any aortic stenosis.
\end{abstract}

Keywords: Aortic dissection, ascending aorta, reinforcement

Battaloğlu B., Akça B., Erdil N., et al. A personalized version of teflon felt sandwich technique for acute type a aortic dissection. EJCM 2017; 05 (1): 07-10. Doi: 10.15511/ejcm.17.00107. 


\section{Introduction}

Type A aortic dissection is a highly life-threatening disease often ending in mortality due to aortic rupture and organ malperfusion. Surgical intervention is the only recommended treatment modality in such cases though it still bears unresolved problems causing mortality and morbidity. Although there is an expanded list of considerable surgical obstacles, aortic root repair remains a challenging problem among these surgical difficulties especially when the aortic dissection extends to the sinuse of valsalva. Furthermore, suturing for the anastomosis of the affected and weakened aortic root due to dissection will cause further damage that might result in uncontrollable surgical bleeding. To solve these problems, numerous valuable surgical techniques were introduced including reinforcement with one or two strips of Teflon felt, glue fixation, supporting partial and entire aortic root with graft, and modifications of the latter. ${ }^{1-7}$

There are, however, some advantages and disadvantages in each of these options. In the traditional felt sandwich technique, the dissected aortic layers are reunited between two strips of Teflon felt (8- to $10-\mathrm{mm}$ wide) provided that the coronary ostia are not compromised. In this study, we present a personalized version of the felt sandwich technique in order to support the entire aortic root externally without causing any aortic stenosis.

\section{Technique}

Following the cardiopulmonary bypass initiation and cross-clamping, an aortotomy is applied and the ascending aorta is transacted just at the supracoranary level. Regardless of the presence of dissection extending to the sinus of valsalva, aortic root is reinforced with the felt sandwich technique in case there is no aortic valve pathology. Unlike the traditional technique, two Teflon strips with non-identical width is prepared; these two strips are approximately 5 -mm wide and $12-$ $\mathrm{mm}$ or wider, respectively (Figure 1A).

Avoiding contact with the origin of the coronary arteries, aortic root is slightly mobilized for the strip application by way of limited excision. The origin of the coronary arteries and surrounding tissues are left untouched in order to prevent potential iatrogenic traumas and further weakening while avoiding strip pressure over the coronary arteries. Then, the wider Teflon strip is placed outside the aortic circumference in order to cover the entire aortic root wall externally. Following this, the $5-\mathrm{mm}$ wide strip is placed inside the aortic circumference. The aortic walls are sandwiched between the felt strips with a horizontal mattress by using 4-0 polypropylene sutures. While placing the horizontal mattress sutures, sutures are applied from the upper side of the outer strip and the mid-point of the inner strip (Figure 1B, Figure 2). While placing the aortic replacement graft, 3-0 polypropylene running sutures
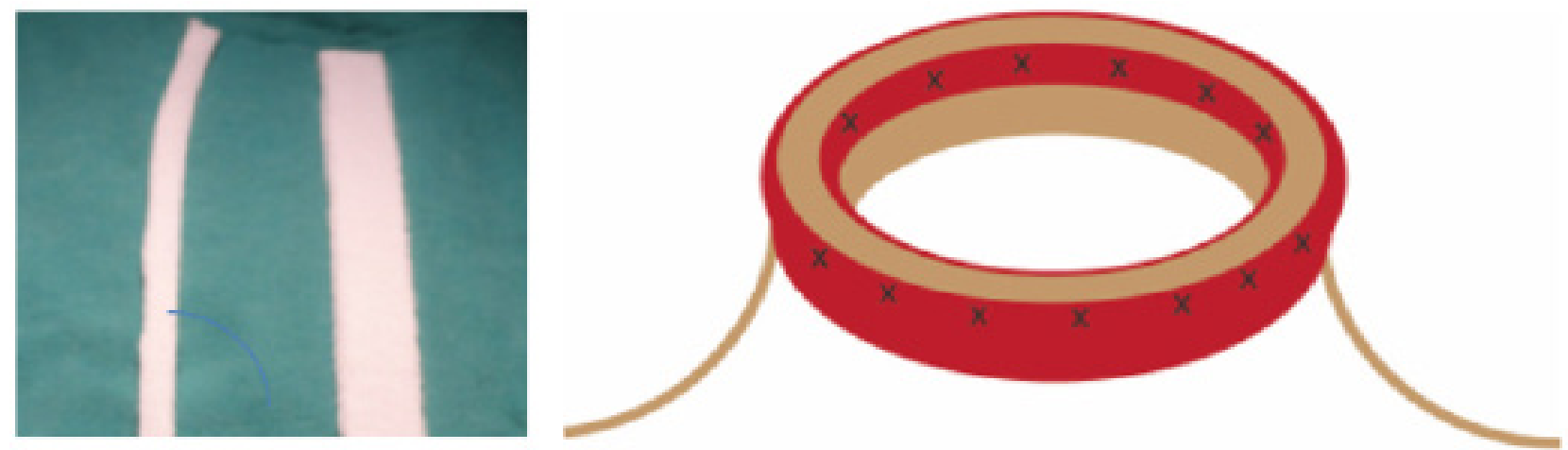

Figure 1: (A) Teflon strips with non-identical; approximately 5-mm wide and 12-mm or wider.

(B) The thin strip is placed inside the aorta and the horizontal mattress sutures are applied from the upper side of the outer strip and the mid-point of the inner strip. 
pass through the bottom line of the inner strip and the brim of the outer strip, which prevents aortic stenosis caused by curled up inner strip (Figure 3A). In other words, if sutures go through the upper or mid-line of the inner strip instead of stabilizing the bottom of the strip, the strip will eventually bend inwards and cause discrete like stenosis (Figure 3B). As the sutures pass through the upper line of the outer strip, the strip is let reach down to the root base. In this way, the strip externally supports the entire aortic root (Figure 3A).

\section{Discussion}

If aortic dissection patients have normal aortic valve with undilated or mildly dilated annulus, surgeons often prefer supracoronary graft replacement due to its technical simplicity and less invasive nature. However, aortic dissection mostly spreads to the aortic root resulting in weakened wall. There may even be additional etiological connective tissue disorders that might further weaken the aortic root wall.
Without one of the reinforcement techniques, proximal anastomosis of this area may cause uncontrollable bleeding and late postoperative aortic root dilatation along with progressive aortic regurgitation. To overcome these problems, many techniques ranging from the simplest methods such as the sandwich technique to more complicated approaches like Florida sleeve technique and its modified versions, which create a neo adventitia, have been suggested to support the aortic root and anastomotic area. ${ }^{1,2}$

Although these techniques offer effective solutions, Florida sleeve technique, its modifications, and similar techniques are time consuming and invasive methods. On the other hand, sandwich technique, simple as it is, may prove to be ineffective as it fails to support the entire aortic root causing surgical bleeding and late root dilatation. Traditionally, the dissected aortic layers are reunited by placing thin felt strips ( 8 - to 10 -mm wide) from inside and outside the circumference of the aorta without

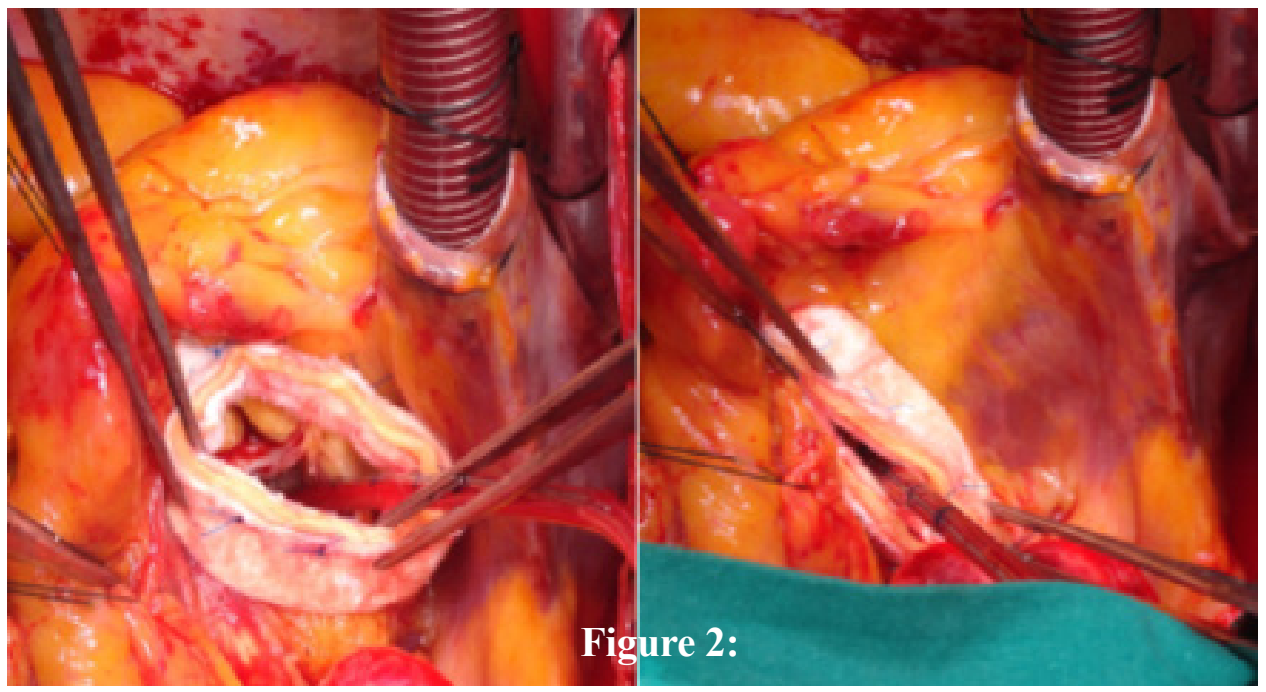

Figure 2: Surgical view of the sandwiched aortic root.

Figure 3: (A) Running sutures pass through the bottom line of the inner strip and the brim of the outer strip and so the outer strip reaches down to the root base. (B) If sutures go through the upper or midline of the inner strip, the strip will bend.

Figure 3:

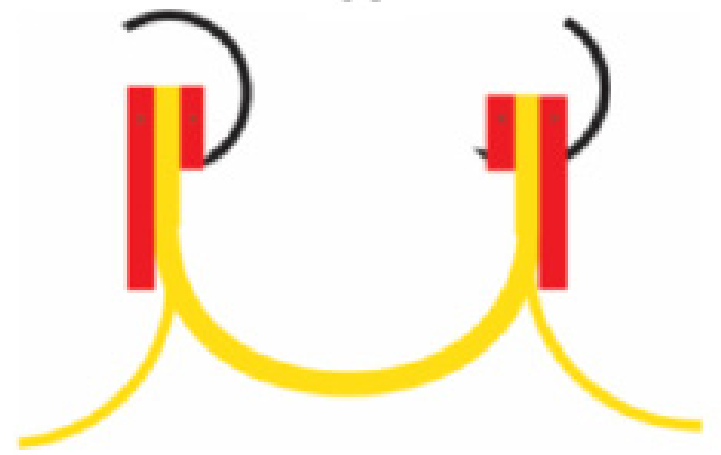

B

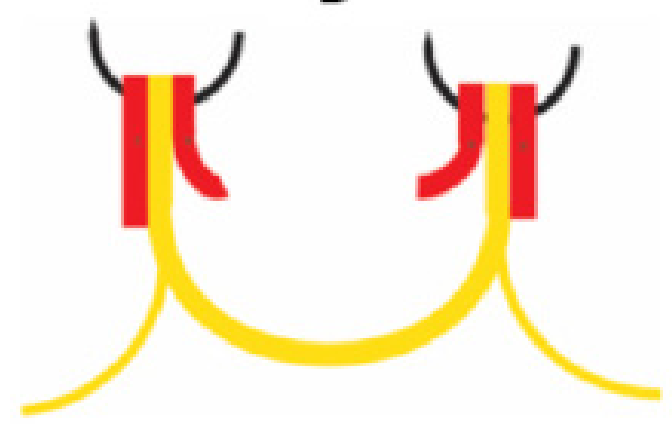


compromising the coronary ostia. In this method, however, the entire aortic root may not be supported.

Furthermore, this method may cause aortic stenosis if running sutures go through the upper or mid-line of the inner strip instead of stabilizing the bottom of the strip, which brings about a curled up inner strip and, therefore, discrete like stenosis. To prevent curling up inner strip I prefer a thinner strip (approximately $5-\mathrm{mm}$ wide) instead of a traditional wider strip and take care to pass sutures through the bottom line of the strip. Safi et al. recommend a technique of interrupted pledgeted horizontal mattress sutures compared to the felt sandwich technique. In their experience, this provides superior stabilization and decreases risk of subsequent aortic stenosis. $^{8}$

\section{References}

1. Hess PJ Jr, Klodell CT, Beaver TM, Martin TD. The Florida sleeve: a new technique for aortic root remodeling with preservation of the aortic valve and sinuses. Ann Thorac Surg 2005;80(2):748-50.

2. Heo W, Min HK, Kang do K, Jun HJ, Hwang YH, Choi JH, Wi JH. A modified root reinforcement technique for acute aortic dissection with a weakened aortic root: a modified Florida sleeve technique and two cases report. J Cardiothorac Surg. 2013;8:203.

3. Chen LW, Wu XJ, Li QZ, Dai XF. A modified valve-sparing aortic root replacement technique for acute type A aortic dissection: the patch neointima technique. Eur J Cardiothorac Surg 2012;42(4):731-33.

4. Urbanski PP, Hijazi H, Dinstak W, DiegelerA. Valve-sparing aortic root repair in acute type A dissection: how many sinuses have to be repaired for curative surgery? Eur J Cardiothorac Surg 2013;44(3):439-44.
In this personalized felt sandwich technique, the aim was to develop the reinforcement effect of the sandwich technique and strengthen the entire aortic root wall externally so as to avoid uncontrollable bleeding and late root dilatation. In this way, the proposed modification also prevents aortic stenosis during surgery. Meanwhile, the original simplicity and applicability of the sandwich technique is preserved as well. This personalized method may also be applied to patients with ascending aortic replacement without dissection disorders.

\section{Conclusion}

The suggested modification to the felt sandwich technique is a simple and effective way of supporting the aortic root externally as it also helps avoid bleeding.

5. Komiya T, Tamura N, Sakaguchi G, Kobayashi T. Modified partial aortic root remodeling in acute type A aortic dissection. Interact Cardiovasc Thorac Surg 2009;8(3):306-9.

6. Nakajima T, Kawazoe K, Kataoka T, Kin H, Kazui T, Okabayashi H, Niinuma $\mathrm{H}$. Midterm results of aortic rapair using a fabric neomedia and fibrin glue for type A aortic dissection. Ann Thorac Surg 2007;83(5):1615-20.

7. Von Oppell UO, Karani Z, Brooks A, Brink J. Dissected aortic sinuses repaired with gelatin-resorcin-formaldehyde (GRF) glue are not stable on follow up. J Heart Valve Dis 2002;11(2):249-57.

8. Safi HJ, Miller CC 3rd, Reardon MJ, Iliopoulos Dc, Letsou Gv, Espada R, Baldwin JC. Operation for acute and chronic aortic dissection: Recent outcome with regard to neurologic deficit and early death. Ann Thorac Surg 1998;66(2):402-11.

Received: 28/11/2016

Accepted: 11/02/2017

Published: 15/03/2017

Disclosure and conflicts of interest:

Conflicts of interest were not reported.

Corresponding author:

Dr. Bektaş Battaloğlu

Mail: bektas.battaloglu@inonu.edu.tr 